\title{
Creep Lifetime Assessment of Pressure-Tight PE100 Pipes Based on a Slow Fatigue Crack Growth
}

\author{
W. B. Luo, ${ }^{\mathrm{a}, \mathrm{b}, 1}$ Y. P. Liu, ${ }^{\mathrm{a}}$ B. Y. Yin, ${ }^{\mathrm{a}}{ }^{\text {Y. }} \mathrm{Li}^{\mathrm{a}}{ }^{\mathrm{a}}$ and X. $\mathrm{Liu}^{\mathrm{a}, \mathrm{b}, 2}$ \\ ${ }^{a}$ College of Civil Engineering and Mechanics, Xiangtan University, Xiangtan, China \\ ${ }^{\mathrm{b}}$ Hunan Key Laboratory of Geomechanics and Engineering Safety, Xiangtan University, Xiangtan, \\ China \\ ${ }^{1}$ luowenbo@xtu.edu.cn \\ 2 liux@xtu.edu.cn
}

Polyethylene pipes are widely used in water supply, gas, and sewage systems due to their excellent mechanical properties. A slow crack growth is the primary fracture mechanism for the pipes under long-term internal pressures. If the creep loading is treated as a special case of fatigue loading, the slow crack growth kinetics of polyethylene is defined in fatigue fracture tests at different stress ratios and extrapolated to creep crack kinetics. Linear elastic fracture mechanics concepts became the basis for predicting the creep lifetime of pressure-tight pipes subjected to various hoop stresses from extrapolated (synthetic) creep crack growth curves, and the prediction is in good agreement with the standard extrapolation, in accordance with ISO 9080.

Keywords: slow crack growth, creep lifetime, stress intensity factor, stress ratio, polyethylene pipe.

Introduction. Polyethylene (PE) is widely used in gas and water supply pipes, due to its excellent mechanical properties. The long-term failure behavior of $\mathrm{PE}$ is of essential for the lifetime and safety assessment of these PE pipes [1-5]. It is generally accepted that the long-term failure behavior of pressurized PE pipes can be separated into three common regions depending on the hoop stress in pipes as illustrated in Fig. $1[1,4]$. In region $A$ for relatively high-stress levels, the pipe failure is mainly caused by the ductile deformation, resulting in a large plastic region. In region $B$ for medium and low hoop stress levels, the pipe failure is manifested as a quasi-brittle slow crack growth (SCG), which is preceded by the crack initiation stage, SCG failures usually happen during a relatively long period. In region $C$, the pipe failure is mainly caused by the aging process, polymer degradation, and stress corrosion cracking, showing stress independence.

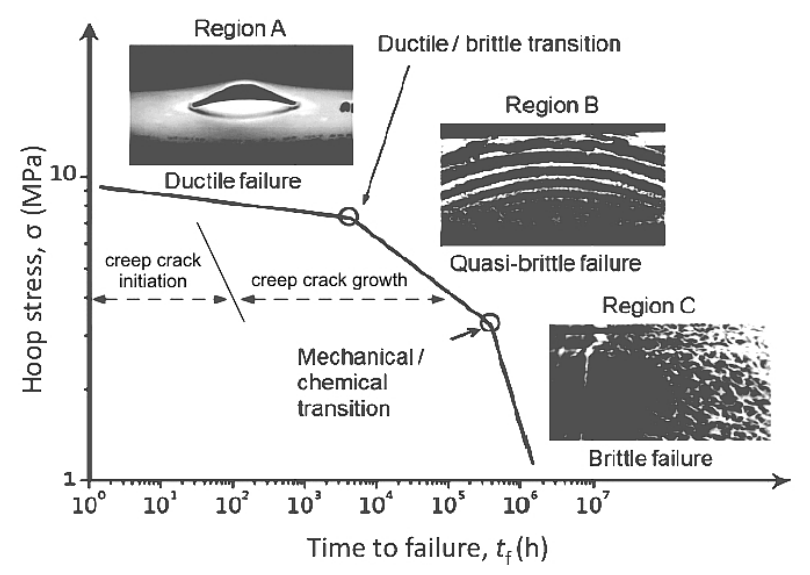

Fig. 1. Schematic illustration of the failure behavior of pressure-tight PE pipes. 
1. Fundamental Model. Taking into account the actual pressure of PE pressure-tight pipe, SCG is the critical failure mechanism of pressurized PE pipes and refers to the most relevant damage in the pipes. Based on this fact, the range of the plastic zone is very small and only presents in the vicinity of the crack tip. Therefore, SCG can be described with linear elastic fracture mechanics (LEFM). In LEFM, the stress distribution near the mode I crack tip is represented by the stress intensity factor $K$ [6]:

$$
K=F \sigma \sqrt{\pi a},
$$

where $\sigma$ is the remote tensile stress, $a$ is the crack length, and $F$ is the dimensionless geometric factor which can be estimated numerically or can be found in the literature for some simple geometries. For a single-edged crack with $a / b \leq 0.6$, in which $b$ is the width of the specimen, whereas the geometric factor $F$ is derived via Eq. (2) [7]:

$$
F=1.12-0.23 \frac{a}{b}+10.55\left(\frac{a}{b}\right)^{2}-21.71\left(\frac{a}{b}\right)^{3}+30.38\left(\frac{a}{b}\right)^{4}
$$

For pipes with an initial crack on the inner wall surface, considering the influence of the crack shape change during the crack propagation, the geometric factor $F$ under internal pressure loading is reduced to the following form [2]:

$$
F=0.3417+0.0588\left(\frac{a}{s}\right)-0.0319\left(\frac{a}{s}\right)^{2}+0.1409\left(\frac{a}{s}\right)^{3},
$$

where $s$ is the pipe wall thickness.

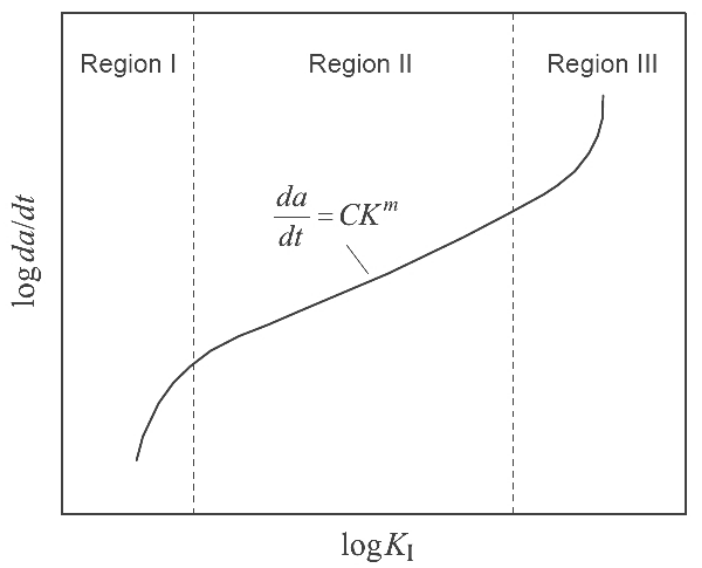

Fig. 2. Scheme of the crack growth rate curve under static loading [2].

The crack growth behavior under static loading, namely creep crack growth (CCG) behavior, of polymer materials, is usually defined by a curve of $d a / d t$ versus $K$ in the double logarithmic coordinate system, as shown in Fig. 2. The curve can be subdivided into three regions: in region $I$, there exists a threshold value for $K$, below which the creep crack is arrested, and for $K$ above the threshold value, the CCG rate decreases with the rising $K$. In region $I I I$, the creep crack grows in an acceleration mode with increased $K$. In region $I I$, the crack exhibits a stable growth: the log-log relationship between $d a / d t$ and $K$ possesses a linear correlation, which can be expressed by the power law of Paris and Erdogan [8]: 


$$
\frac{d a}{d t}=C K^{m},
$$

in which, the parameters $C$ and $m$ are material constants.

According to creep and long-term fracture approach, the crack growth process under static loading is a very long-term period ranging from several years to several decades. Thus, an accelerated characterization method is necessary [9-12]. According to [1], fatigue crack growth (FCG) tests under various stress ratios can be used to quickly predict the creep failure lifetime of PE pipes under static loading as illustrated in Figs. 3 and 4. The critical point in this extrapolation approach is that the creep loading of a material can be considered as a special case of fatigue loading when the stress ratio $R=1$. In Fig. 3, for the fatigue tests of PE specimen with an initial crack, $R$ is the ratio of the minimum load to maximum one. In the case of static loading condition, $R$ is equal to 1 . According to the extrapolation methodology in Fig. 4, the FCG curves at different $R$ are acquired through corresponding fatigue tests (left diagram) and transformed into the $K_{\max }-R$ curves (right diagram); then, the maximum stress intensity factors at various constant crack growth rates are extended to $R=1$ (static loading) via appropriate mathematical methods (right diagram); finally, the extrapolation results are transformed back into the kinetics diagram to generate a "synthetic" CCG curve (left diagram). Furthermore, the material parameters $C$ and $m$ under static loading can be determined and used for lifetime prediction of pressure-tight pipes using Eq. (5) by considering the relevant stress intensity factors of a growing crack in a pipe with an initial crack on its inner surface:

$$
t_{f}=\int_{a_{0}}^{a_{f}} \frac{1}{C K^{m}} d a,
$$

where $a_{0}$ is the initial crack length and $a_{f}$ is the crack length at failure.

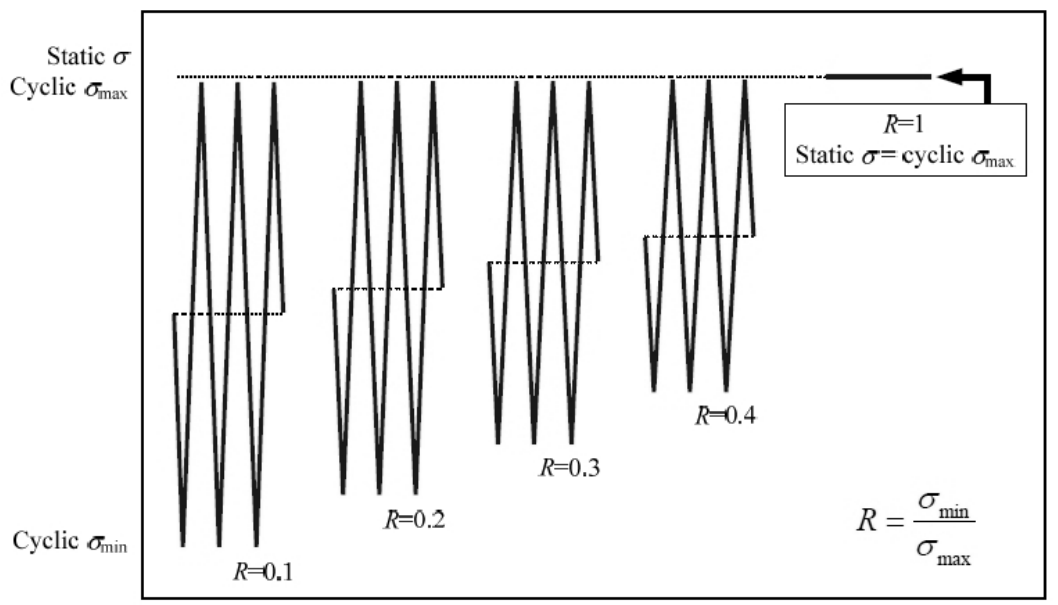

Fig. 3. Crack tip stress field for static and cyclic loads at various $R$-ratios [1].

2. Experiment. The dumbbell-shaped tensile specimens with a $2 \mathrm{~mm}$ long prefabricated crack are cut from the PE100 pipes for water supply, which are provided by Akan Enterprise Group (Shanghai) Co. Ltd. The shape and geometrical dimensions of the specimen are shown in Fig. 5, and the specimen thickness is $4 \mathrm{~mm}$. Fatigue tests are carried out on a CARE-M3000 dynamic electromagnetic force test machine at $20^{\circ} \mathrm{C}$ (see Fig. 6). A 


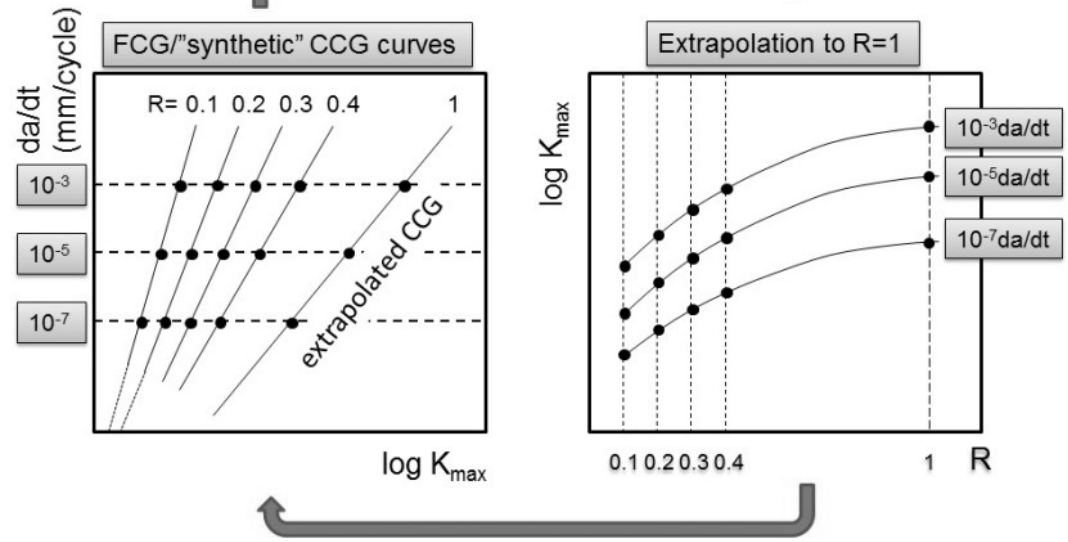

Fig. 4. Methodology to generate synthetic SCG curves for static loading based on cyclic tests [1].

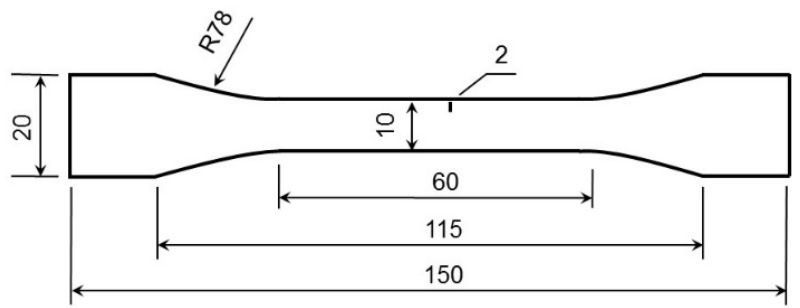

Fig. 5. The geometry of the test specimen (unit: $\mathrm{mm}$ ).

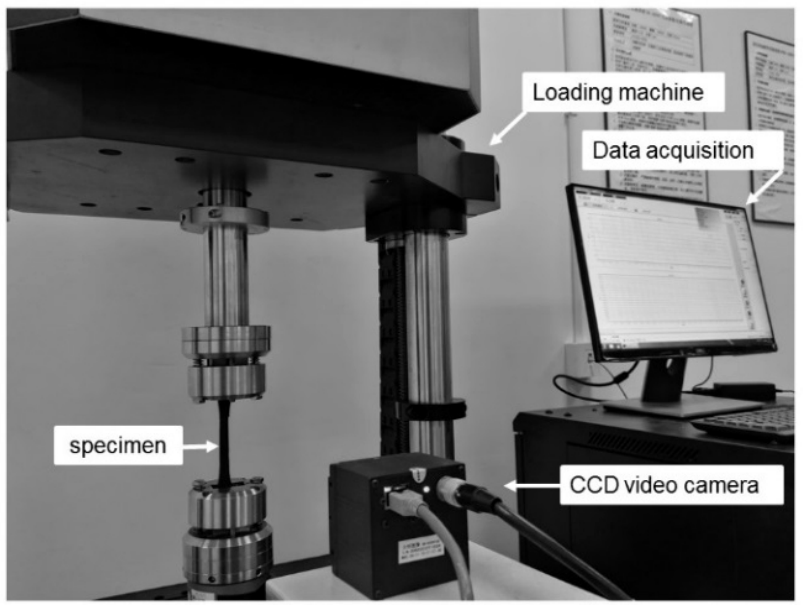

Fig. 6. Experiment system.

sinusoidal tension is applied to specimens under a force-controlled mode with a maximum tensile force of $500 \mathrm{~N}$, namely, $12.5 \mathrm{MPa}$, and various stress ratios $(R=0.1,0.2,0.3$, and $0.4)$ at a frequency of $8 \mathrm{~Hz}$. The crack profiles are sequentially recorded in the real time-scale by a CCD camera and automatically identified by a MATLAB image processing program developed by the authors. Figure 7 shows an example of the FCG process for $R=0.1$ and the corresponding crack tip/lip images, with which the crack lengths and growth rates are determined by the image processing. 
3. Results and Discussion. It can be seen from Fig. 7 that the crack grows gradually and then suddenly loses its stability at some instant when the crack grows to approx. a half width of the specimen, resulting in the total fracture. The fatigue lifetime $t_{f}$ and crack length $a_{f}$ at fracture are listed in Table 1 . The time to failure increases markedly with the ascent of $R$-ratio. Nevertheless, it is noteworthy that the crack-tip plastic zone is very small so that the LEFM is applicable for the crack growth analysis.

$\mathrm{T}$ a b 1 e 1

The Fatigue Lifetime and the Crack Length at Fracture

\begin{tabular}{|c|c|c|}
\hline$R$ & $t_{f}, \mathrm{~s}$ & $a_{f}, \mathrm{~mm}$ \\
\hline 0.1 & 14,790 & 3.9754 \\
\hline 0.2 & 24,960 & 3.7983 \\
\hline 0.3 & 84,990 & 4.0554 \\
\hline 0.4 & 170,520 & 2.9426 \\
\hline
\end{tabular}

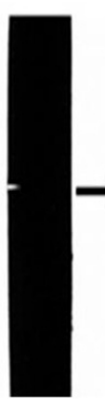

a

360 s

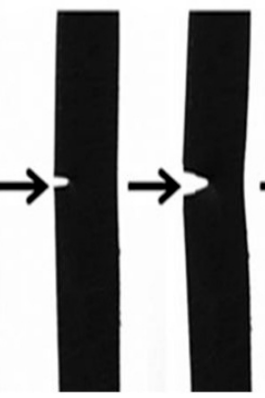

b

13500 s

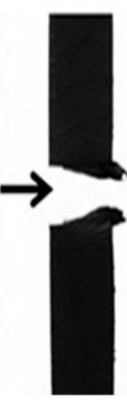

d

$14851 \mathrm{~s}$

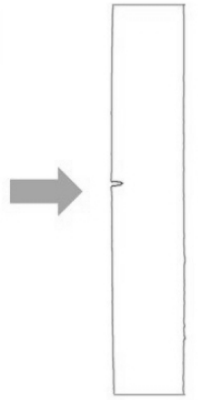

a'

360 s

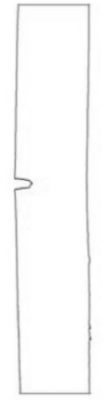

b'

13500s

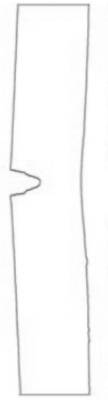

C' d'

14850s 14851s

Fig. 7. FCG process and the corresponding crack tips/lips for $R=0.1$.

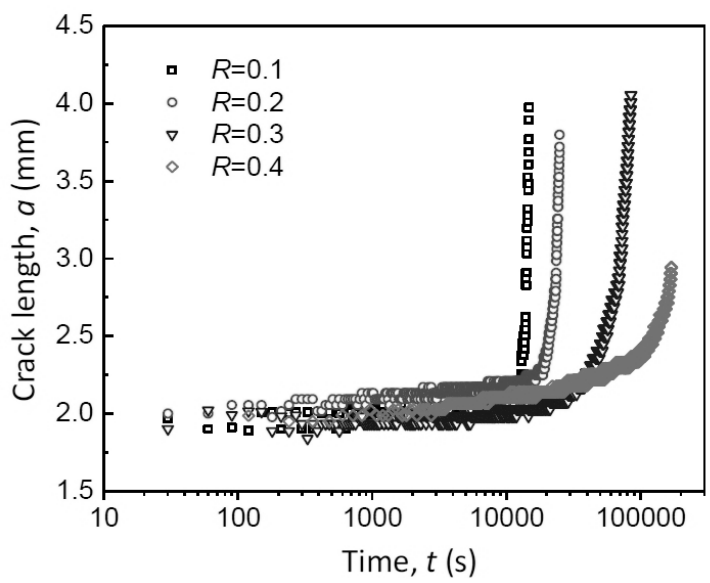

Fig. 8. FCG curves for various stress ratios $R$.

Figure 8 presents the fatigue crack length variation with time for $R$-ratios of $0.1,0.2$, 0.3 , and 0.4 , respectively. It can be also seen in Fig. 8 that the crack grows very slowly and the shape of curves is just like a "stair" at the initial stage. It is obvious that the FCG is 
discontinuous, which reveals that there is an incubation time for crack nucleation and merging before the crack growth. After about $10,000 \mathrm{~s}$ or 80,000 cycles, the cracks begins to grow more and more rapidly until a sudden failure.

From Fig. 8, crack growth rates $(d a / d t)$ are calculated, and the corresponding stress intensity factors $\left(K_{\max }\right)$ are determined via Eqs. (1) and (2). Figure 9a shows the $d a / d t$ versus $K_{\max }$ curves for $R$-ratios of $0.1,0.2,0.3$, and 0.4 . It is seen that for a given $K_{\max }$, the crack growth rate decreases with the ascent of $R$-ratio. The obtained crack kinetic curves in region $I I$ demonstrate that the relationship of $d a / d t$ versus $K_{\max }$ is approximately linear in the log-log scale, following the power via Eq. (4). Hence, these crack kinetic curves in Fig. 9a are linearly extrapolated to higher $K_{\max }$ for each $R$-ratio, and the $K_{\max }$ values at given crack growth rates, such as $10^{-5}, 10^{-4}$, and $10^{-3} \mathrm{~mm} / \mathrm{s}$, are obtained for each $R$-ratio and plotted in Fig. 9b. It is obvious that the $K_{\max }$ needed to keep constant crack growth rate increases linearly with the rising $\log R$, and the $K_{\max }$ values for the three constant crack growth rates at $R=1$ are determined by extrapolation. These extrapolation data are then re-plotted in Fig. 9a to produce the synthetic crack growth kinetics, namely the CCG law. It can be seen that the extrapolations are in good agreement with the power law of Eq. (4) with $C=1.92 \cdot 10^{-7}$ and $m=3.61$.

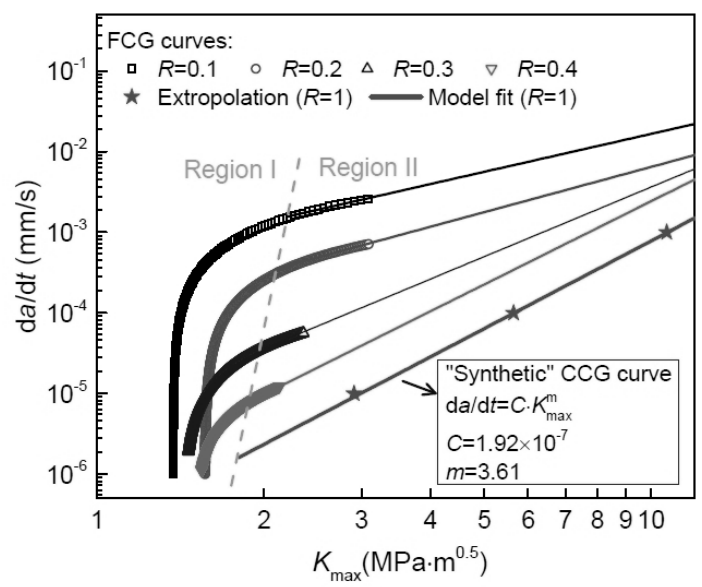

a

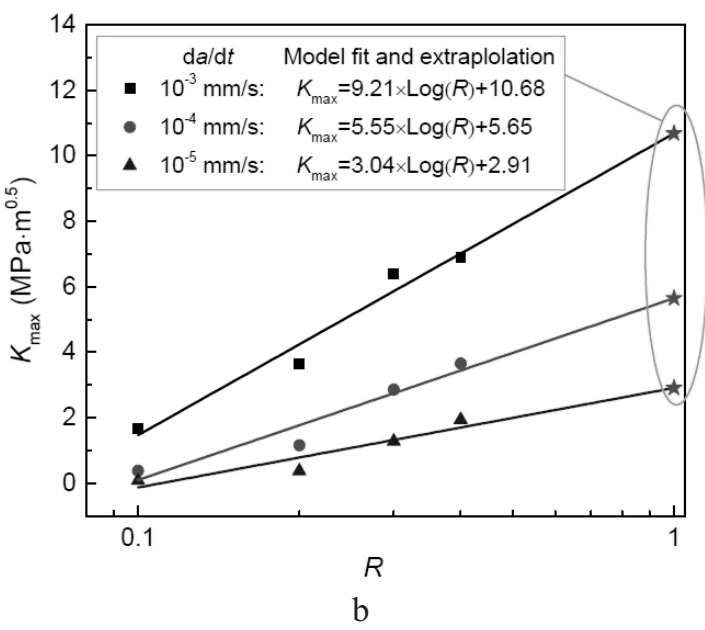

Fig. 9. Crack kinetics of PE100 at different $R$-ratios and extrapolation of "synthetic" CCG curve (a); extrapolation of $R$-ratio dependence of SIF to $R=1.0$ (b). 
The creep failure time of the pressure-tight pipe is estimated for various hoop stress values, as shown in Fig.10. Since there are no experimental failure time data for hoop stresses below $10 \mathrm{MPa}$ at room temperature due to the long time expectation up to several decades, Fig. 10 only shows the long-term hydrostatic strength data, which exhibit a ductile failure mode (Region $A$ ), up to $10^{4} \mathrm{~h}$. These were obtained from the internal pressure tests. According to ISO 9080, the standard extrapolation method based on time-temperature superposition is used to predict the ductile-brittle transition knee and the quasi-brittle failure time under SCG mechanism (Region $B$ ), as shown by dashed lines in Fig. 10. The prediction of the failure time via Eqs. (3) and (5) with two determined parameters $C$ and $m$ is also given by the solid line in Fig.10. Here, a PE100 pipe with standard dimension ratio (SDR) of 11 and diameter of $160 \mathrm{~mm}$ is considered, and the typical size of the initial crack $a_{0}$ in pipe is preset to be $0.1 \mathrm{~mm}$, as observed experimentally in [4]. The predictions in Fig. 10 for PE100 at $20^{\circ}$ by the proposed method based on the LEFM concept and FCG accelerated characterization are in good agreement with those provided by ISO 9080 standard extrapolation method.

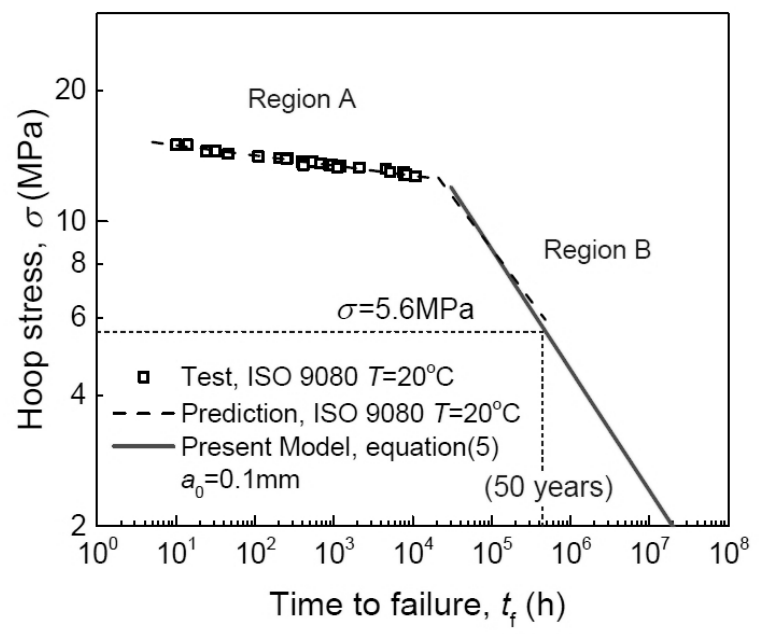

Fig. 10. Hoop stress vs. time to failure curves determined via internal pressure tests (dots) and predicted via ISO9080 (dashed line) and proposed method (solid line) for PE100 pipes at $20^{\circ} \mathrm{C}$.

Conclusions. In this study, the crack growth kinetics of PE material is investigated under cyclic loads at various $R$-ratios. For the determination of the crack growth kinetics, a method of measuring crack length is developed based on digital image technology. An extrapolation concept for the assessment of CCG is applied for one kind of PE pipe. With the "synthetic" CCG curve, lifetime prediction based on LEFM is possible. The result of this study shows the residual service life of a PE pipe with an initial $0.1 \mathrm{~mm}$-long crack is estimated to exceed 50 years when the hoop stress is lower than $5.6 \mathrm{MPa}$, and the validity of the proposed extrapolation method is verified.

Acknowledgments. This work was supported by the National Natural Science Foundation of China (11572275) and the Hunan Provincial Innovation Foundation for Postgraduate, China (CX2017B309).

1. A. Frank, W. Freimann, G. Pinter, et al., "A fracture mechanics concept for the accelerated characterization of creep crack growth in PE-HD pipe grades," Eng. Fract. Mech., 76, 2780-2787 (2009).

2. P. Hutar, M. Sevcik, L. Nahlik, et al., "A numerical methodology for lifetime estimation of HDPE pressure pipes," Eng. Fract. Mech., 78, 3049-3058 (2011). 
3. A. Frank, I. J. Berger, F. Arbeiter, et al., "Lifetime prediction of PE100 and PE100-RC pipes based on slow crack growth resistance," in: Plastic Pipes Conference PPXVIII, Berlin (2016), DOI: 10.13140/RG.2.2.27467.80165.

4. R. W. Lang, A. Stern, and G. Doerner, "Applicability and limitations of current lifetime prediction models for thermoplastics pipes under internal pressure," Macromol. Mater. Eng., 247, 131-145 (1997).

5. N. Merah, Z. Khan, A. Bazoune, et al., "Temperature and loading frequency effects on fatigue crack growth in HDPE pipe material," Arab. J. Sci. Eng., 31, 19-30 (2006).

6. A. Frank, P. Hutar, and G. Pinter, "Numerical assessment of PE 80 and PE 100 pipe lifetime based on Paris-Erdogan equation," Macromol. Symp., 311, 112-121 (2012).

7. H. Tada, P. C. Paris, and G. R. Irwin, The Stress Analysis of Cracks Handbook, 3rd edn, ASME Press, New York (2000), p. 53.

8. E. Nezbedová, G. Pinter, A. Frank, et al., “Accelerated tests for lifetime prediction of PE-HD pipe grades,” Macromol. Symp., 373, 1600096 (2017).

9. G. Pinter, M. Haager, W. Balika, and R. W. Lang, "Cyclic crack growth tests with CRB specimens for the evaluation of the long-term performance of PE pipe grades," Polym. Test., 26, 180-188 (2007).

10. A. Frank, G. Pinter, and R. W. Lang, "A novel qualification procedure for lifetime and safety assessment of PE pressure pipes for arbitrary installing conditions," in: Plastic Pipes Conference XIV, Budapest (2008).

11. R. W. Lang, G. Pinter, and W. Balika, "Konzept zur Nachweisführung für Nutzungsdauer und Sicherheit von PE-Druckrohren bei beliebiger Einbausituation," 3 R International, 44, 33-41 (2005).

12. V. Favier, T. Giroud, E. Strijko, et al., "Slow crack propagation in polyethylene under fatigue at controlled stress intensity," Polymer, 43, 1375-1382 (2002).

Received 05. 03. 2018 